\title{
Capital Social y MERCADOS FINANCIEROS CREDITICIOS: DEMANDA DE CRÉDITO EN MÉXICO, 2010
}

\author{
Ernesto Zepeda, Juan Antonio Leos y Félix Carvallo
}

Fecha de recepción: 10 de abril de 2015. Fecha de aceptación: 24 de agosto de 2015.

\begin{abstract}
RESUMEN
En presencia de fallas de mercado, las personas necesitan encontrar mecanismos aceptables que permitan reducir la incertidumbre y maximizar su posible beneficio. Como la principal falla es la asimetría de la información existente, las personas recurren a canales de transmisión de información que compensen las fallas. Estos canales son las redes de relaciones sociales. Esto es el Capital Social, la red de personas que un individuo posee, y cuyo uso puede traerle beneficios. En el caso del mercado financiero (crediticio en especial), las personas hacen uso del Capital Social antes de tomar decisiones dentro del mercado. Mediante una serie de modelos lineales, tomando información de la wvs-2010 y ENIGH-2010, se encontró que la demanda de crédito responde a algunas variables que muestran la percepción de los individuos sobre su entorno (información).
\end{abstract}

Palabras clave: capital social, créditos, mercados financieros, servicios financieros, modelos lineales.

Clasificación JEL: C35, E44, G2, O16, P43.

\section{Social Capital and Financial Credit Markets: Credit Demand in Mexico, 2010}

\begin{abstract}
In the presence of market failures, people need to find acceptable mechanisms to reduce uncertainty and maximize potential benefits. Because the principal failure is the asymmetry of information available, people resort to channels that transmit information to compensate for these drawbacks. These channels are networks of social relationships. This phenomenon is referred to as Social Capital, the network of people that an individual possesses, and whose use could bring about benefits. In the case of the financial market (especially the credit market), people make use of Social Capital when making decisions within the market. Through a series of linear models, drawing on data from wvs-2010 and ENIGH-2010, it was found that credit demand responds to a few variables that indicate individuals' perceptions of their environment (information).
\end{abstract}

Key Words: social capital, credit, financial markets, financial services, linear models. 


\section{CAPITAL SOCIAL ET MARCHÉS FINANCIERS CRÉDITEURS: DEMANDE DE CRÉDIT AU MEXIQUE, 2010}

\section{Résumé}

Face à des déficiences du marché, les gens ont besoin de trouver des mécanismes acceptables qui permettent de réduire l'incertitude et de maximiser leur possibilité de bénéfice. Comme la principale déficience est la diversité de l'information existante, les personnes recourent à des canaux de transmission d'information qui compensent les déficiences. Ces canaux sont les réseaux de relation sociale. Ceci est le capital social, le réseau de relations personnelles dont dispose un individu a et dont l'usage peut lui générer des bénéfices. Dans le cas du marché financier (créditeur en particulier), les gens font usage du capital social avant de prendre des décisions vis-à-vis du marché. A travers une série de modèles linéaires, en prenant en compte des informations de la wVs-2010 et ENIGH-2010, il a été constaté que la demande de crédit répond à certaines variables révélatrices de la perception qu'ont les individus de leur environnement (information).

Mots clés: capital social, crédit, marchés financiers, services financiers, modèles linéaires.

\section{CAPITAL SOCIAL E MERCADOS FINANCEIROS CREDITÍCIOS: DEMANDA DE CRÉDITO NO MÉXICO, 2010 \\ Resumo}

Na presença de falhas de mercado, as pessoas necessitam encontrar mecanismo aceitáveis que permitam reduzir a incerteza e maximizar o seu possível benefício. Como a principal falha é assimetria da informação existente, as pessoas recorrem a canais de transmissão de informação que compensem estas falhas. Estes canais são as redes de relaçóes sociais. Este é o Capital Social, a rede de personas que um indivíduo possui, e cujo uso pode lhe trazer benefícios. No caso do mercado financeiro (creditício em especial), as pessoas usam o Capital Social antes de tomar decisóes dentro do mercado. Através de uma série de modelos lineares, tomando informação da wvs- 2010 e ENIGH-2010, se encontrou que a demanda de crédito responde a algumas variáveis que mostram a percepção dos indivíduos sobre o seu entorno (informação).

Palavras-chave: capital social, créditos, mercados financeiros, serviços financeiros, modelos lineares.

\section{社会资本和信贷金融市场：2010年墨西哥信贷需求}

摘要

面对市场失灵，人们必须找到其他方法以减少不确定性并将可能受益最大化。由于 市场最主要的失灵在于现存信息的不对称性, 人们便寻求其他信息传递的渠道以弥 补市场失灵。这些渠道就是社会关系网络, 即社会资本, 也就是一个人所拥有的人 脉资源。人脉的运用能够带来利益。就金融市场（尤其是信贷市场）而言, 人们在 做决定前通常会运用他的社会资本。通过一系列线性模型, 且将2010年WVS模型和 2010 年ENIGH模型的信息纳入考虑后, 人们发现, 信贷需求与某些变量相关, 而这 些变量反应了个人对其周围信息环境的认识。

关键词：社会资本，信贷，金融市场，金融服务，线性模型 


\section{INTRODUCCIÓN}

En México, el uso de los servicios financieros es escaso con relación a la parte de la población que podría hacer uso de ellos. El ahorro bancario $(18.0 \%$ de la población) y la demanda de créditos $(14.0 \%)$ son menores a $1 / 5$ de la población con posibilidades de ahorro derivados de un nivel de ingresos suficientes para esta actividad. Se trata de un mercado no desarrollado y con distintas dificultades para su acceso.

No obstante, la necesidad de financiamiento y ahorro son fundamentales en la vida de las personas y, hasta donde se conoce, se puede satisfacer. Sin embargo, no es mediante el uso de instrumentos formales que se hace, pues gran parte de la población $(75.0 \%)$ prefiere acudir a familiares y conocidos, ya que les genera mayor confianza o comodidad, aunque no sea lo económicamente óptimo.

Algunas de las razones de esto son las fallas del mercado financiero, entre las principales están: la falta de información aceptable (Stiglitz, 1989), la poca confianza en los bancos y el anonimato de los agentes dentro del mercado (DiGiannatale et al., 2008). Sin embargo, la obtención de créditos ocurre. Son prestamistas o familiares quienes transfieren parte de sus ahorros a quienes los solicitan, a cambio de pago de intereses o pagos de otro tipo, ya sea en especie o trabajo. Es decir, las personas hacen uso de la red de conexiones interpersonales que poseen $y$, mediante este tráfico de información, acceden a oportunidades que les reportan beneficios tangibles. Es decir, las personas hacen uso de su Capital Social (Cs).

El cs es un concepto complejo, difícil de definir, medir o probar por su carácter social. Sin embargo, es tangible en la vida cotidiana de la sociedad e incluso en el mercado. No obstante, el poder de dicho capital es manejado por fuera del sistema económico, ya que sirve como un medio de corrección de la información con que cuentan las personas y, por tanto, es un referente en su toma de decisiones en la realidad. También sirve para reflejar el grado de desconfianza que los individuos pueden tener hacia los mecanismos formales de financiamiento.

En el caso de México, se encontró que el cs en el año 2010 fue difícil de medir por medios tradicionales, pero pudo observarse su influencia directamente con el uso de variables estratégicas dentro de modelos econométricos. De esta forma, la desconfianza en los bancos, o la preferencia de sistemas informales, representaron las principales variables que pueden explicar la estructura de la demanda de crédito, y la dinámica interna del sistema financiero mexicano en general. 


\section{JUSTIFICACIÓN}

Los cambios en la estructura de los mercados sigue siempre (y viceversa) a los cambios de la sociedad, es decir, a los cambios en la conducta de las personas. En la economía, las decisiones se basan en las expectativas de las personas, quienes dependen de la información a su alcance para realizar procesos racionales previos a su toma de decisiones. Por esto, el cs puede ayudar a explicar algunas relaciones económico-sociales, así como su desarrollo. Dado que el mercado financiero afecta a los sectores productivos, su estudio es estratégico para la planeación económica de las naciones; en especial el sector primario, $y$, por ende, para los productores que utilizan los recursos naturales directos.

\section{PLANTEAMIENTO DEL PROBLEMA}

Dado que el crédito es parte del engranaje económico, comprender sus distorsiones es fundamental para lograr encaminar el desarrollo económico a todo el sistema de manera funcional; además, puede ayudar a determinar la salud del sistema financiero completo. En México, el crédito no es un servicio distribuido universalmente, considerando los montos que reporta oficialmente. Para entender cómo las personas deciden acudir al mercado o no, es necesario comprender algunos de los factores en los que se basan y estudiar si eso afecta sus decisiones dentro del mercado. El cs es una herramienta que no sólo describe el comportamiento de los individuos, sino que ayuda a las personas a tomar decisiones óptimas, dadas sus circunstancias específicas. En pocas palabras, el cs es la red de relaciones humanas de un agente específico, y el flujo de información útil que le otorga mantener dicha red.

En el sistema financiero el Cs puede usarse para comprender el grado de formalidad institucional al que las personas prefieren acudir, así como el nivel de riesgo que están dispuestas a tomar. Es necesario plantearse cuál es la naturaleza de la relación del cs y la dinámica interna de los mercados de servicios financieros, específicamente, el de crédito.

\section{OBJETIVOS E HIPÓTESIS}

El objetivo central de esta investigación fue comprender cómo y de qué manera afectó el cs en México, durante el 2010, la dinámica del mercado de servicios financieros y crédito, de modo que se puedan hacer recomendacio- 
nes sobre el fenómeno que sean útiles a los diseñadores de políticas públicas. Como objetivo particular se estableció comprender el concepto de Cs y su relación con el sistema financiero para poder definir un modelo cuantitativo que midiera el fenómeno. A través de un grupo de 11 modelos se estudiaron las determinantes del problema, contrastando teóricamente los datos reportados oficialmente por el INEGI. ${ }^{2}$

Las hipótesis planteadas se centraron en que el concepto de cs es un concepto que puede explicar las fallas del mercado financiero, y que de hecho guarda relación con la demanda de crédito que efectúan los agentes. También se consideró que la distribución funcional de información puede permitir corregir el mercado. Con estos resultados se puede discutir un análisis de política pública que incluya la percepción de las personas como un indicador de corrección en la toma de sus decisiones económicas.

\section{DISCUSIÓN TEÓRICA}

Las redes sociales son los vínculos que existen entre los miembros de un grupo, o sociedades que guardan una relación de conocimiento mutuo. Las redes son el entramado que explica cómo las personas interaccionan, las condicionantes para lograr la convivencia y sus obligaciones, ya que son relaciones no triviales. El cs permite acercarse a esta idea, ya que se puede usar para medir su impacto en fenómenos socioeconómicos (Alarcón et al., 2003; DiGiannatale et al., 2008; Mitchell et al., 1997).

El cs es un recurso que los individuos usan para acceder a oportunidades que les resulten estratégicas, con la característica de que se crea, acumula y refuerza en procesos históricos lentos. El cs se construye, destruye y renueva periódicamente, apegado a costumbres y experiencias, lo que genera y oferta el uso de sus relaciones. Aplicada a la economía, permite la reducción de los costos de transacción e información. El cs es un concepto multidimensional, cognoscitivo y estructural que depende de variables socioeconómicas y cívicas (Boix y Posner, 1996; Bourdieu, 1985; Espinoza y Rabi, 2009; Neira, 2002; De la Torre et al., 2011).

Neira (2002) propone lo siguiente: 
Ernesto Zepeda, Juan Antonio Leos y Félix Carvallo

Cuadro 1. Definiciones básicas del Capital Social en el tiempo

\begin{tabular}{lll}
\hline \multicolumn{1}{c}{ Autor } & \multicolumn{1}{c}{ Elementos generadores } & \multicolumn{1}{c}{ Consecuencias } \\
\hline Bourdieu (1985) & $\begin{array}{l}\text { Redes permanentes y la pertenencia a un } \\
\text { grupo. }\end{array}$ & $\begin{array}{l}\text { Que aseguran a sus miembros un conjunto } \\
\text { de recursos actuales o potenciales. }\end{array}$ \\
Coleman (1988) & Aspectos de la estructura social. & Que facilitan ciertas acciones comunes de \\
los agentes dentro de la estructura.
\end{tabular}

*Social Capital Interest Group. Michigan University.

Fuente: Neira (2002).

Putnam $^{3}$ (1993) presenta otra visión, donde el cs representa la oportunidad de alcanzar objetivos específicos por un grupo social, aunque ha sufrido una fuerte crítica. El desarrollo territorial es un fenómeno complejo de carácter sistémico, en el cual la confianza y las relaciones sociales son la clave (Boisier, 2003). Igualmente, el desarrollo territorial requiere de un contexto de descentralización institucional. Coleman (1988) afirma que el cs reside en las instituciones de manera natural, y por tal, en todas las instituciones, y dentro de la racionalidad, y las afecta. Entre más desigual es una sociedad, más débil es el cs. Otro problema es la fuerza relativa horizontal, debido a intereses localizados, o contraer obligaciones morales más fuertes. El cs puede ser integrado 
a la teoría económica debido a que ayuda a comprender la estructura de expectativas de los individuos, así como sus criterios en la toma de decisiones, que normalmente no son contabilizados por los estrechos criterios de racionalidad de la teoría económica formal. ${ }^{4}$ Minimizar el costo de transacción depende de reglas legales y formales, garantía de propiedad privada, y normas sociales (Alarcón et al., 2003; Alex Alarcón et al., 2003; Barro, 1996; Boix y Posner, 1996; Espinoza y Rabi, 2009; Figueroa y Cázarez, 1999).

Los stakeholders ${ }^{5}$ actúan fuera de las instituciones formales y son agentes que toman decisiones en mercados informales, e incrementan el costo de transacción de todas las actividades sociales. Pero pueden ser absorbidos por las instituciones formales, ya que el costo de la falla del mercado es superior a la integración. Todos los defectos del cs actúan como multiplicadores de los costos de transacción, pero sus beneficios los contrarrestan; se trate de bienes públicos o privados, incluso con distorsiones tipo free-rider (Boix y Posner, 1996; Jones y Hill, 1988).

Existen tres niveles de relaciones sociales básicas: bonding, bridgingy linking. Aunque se pueden agregar dos más: sinérgico y económico. Estos representan la forma en que las personas interactúan, y el grado de cercanía y utilidad, así como la afectación mutua. Cada nivel es un grado de interrelación que genera distintos grados de bienestar, o fronteras, maximizando ciertas oportunidades. Se entiende que se le considere una forma de capital. Aunque bien pueden ser coercitivos, su racionalidad interna les ofrece distintas posibilidades extraordinarias a las comunes. También son posibles algunos problemas asociados, tales como exclusión, corrupción, ligas criminales, autoritarismo, etcétera, e intereses contrarios a los sociales (Alex Alarcón et al., 2003; Boix y Posner, 1996; Dobruck Lowe, 2012; Espinoza y Rabi, 2009; DiGiannatale et al., 2008; Neira, 2002; Mitchell et al., 1997; De la Torre et al., 2001).

Las fallas de mercado son perturbaciones que afectan su funcionamiento. La mayor parte de ellas ocurren en un nivel superior, dado que no se limita al acceso al mercado, distribución de información de calidad y organización de los agentes, sino que se ve afectado por las capacidades del capital humano. Las fallas del mercado financiero son de información, en la solidez institucio-

4 Es decir, se puede entender cómo perciben las personas a otras personas para determinar decisiones que aunque no son económicamente racionales, sí encuentran un óptimo delimitado por otros costos que no son contabilizados: como la confianza institucional, la percepción de justicia, y la confianza en los productos ofertados formal o informalmente.

5 Análisis de involucrados (Freeman, 1988; citado por Mitchell et al., 1997). Los stakeholders son los tomadores de decisiones en los mercados. 
nal y la capacidad de los propios agentes de enfrentar al mercado. Otras perturbaciones son el nivel de precios, la competencia imperfecta, la corrupción, la geografía de la información, la capacidad de aprendizaje de las personas, el equilibrio de mercado, la histéresis, la dinámica económica, y las externalidades, entre otros.

En México, la deficiencia de educación es crucial para entender que la racionalidad de los agentes está afectada por su umbral educativo. La información imperfecta causa una selección adversa (calidad de carteras), un riesgo moral (desvío del fin del crédito), y el forzamiento de las obligaciones crediticias. Por lo tanto, los agentes son cautelosos con sus decisiones, tratando de llenar los huecos en la información por todos los medios (DiGiannatal et al., 2008; Sokol, 2007; Stiglitz, 1989).

El anonimato, característica de los mercados desarrollados, es el desconocimiento entre agentes dentro del mercado; su relación se limita al mercado. La ventaja es que los agentes participan sólo por la persecución de sus intereses, y no por intervención de otros agentes. El anonimato es condición de infinitud en el mercado, donde se guían por la información, y cuyos intereses no afectan los procesos de mercados eficientes. Esto genera cierto recelo, o desconfianza a algunos agentes. En presencia, o expectativas de falta de transparencia, o protección legal, los agentes tardan en emitir sus juicios, y decidir. La falta de transparencia en los mercados, procesos, leyes, reglamentos, agentes, etcétera, fomenta la desconfianza, lo que provoca que los individuos acudan a instituciones financieras menos formales, pero más conocidas, claras o relativamente menos costosas (DiGiannatale et al., 2008), al menos en su perspectiva.

Un concepto de poco uso en el estudio del cs es el de la libertad. La libertad es la capacidad de los individuos de elección y acción, sujetos a las normas, además de ser el centro de desarrollo de la sociedad, es decir, el sistema institucional. La violación garantiza un castigo. En la economía, el concepto es semejante. Parte de las características de esa libertad se reflejan en la capacidad de acceder o proporcionar servicios económicos. Por ello, se considera que el PIB $^{6}$ o el ingreso per cápita pueden ser medidas usadas para expandir la libertad de la sociedad (elección, producción y consumo) mediante su comprensión en el desarrollo de políticas públicas, pero no son determinantes si no se contabilizan a través de la racionalidad de las personas. En el caso de los mercados financieros, el nivel de acceso libre a los servicios y a los productos financieros, el costo relativo de transacción o la exclusión, son barreras a la 
libertad. Si los agentes no tienen acceso a los servicios financieros formales, su libertad económica no se cumple. Por lo que el sistema económico no se desarrolla de forma correcta, y genera fallas, que normalmente son costosas para la misma sociedad y el mercado. La deficiencia al acceso de crédito vulnera la productividad y el consumo, lo que daña a la sociedad. Más aún, cuando está condicionado por factores que parten de la forma de interrelaciones sociales, el cs es explicativo de la naturaleza de los actos de los individuos. La estructura del cs modela la naturaleza de las relaciones económicas de los agentes, que está acotada por el grado y tipo de libertades que poseen (Sen, 2000; Mill, 1859).

La inclusión financiera se encuentra interrelacionada con la estabilidad financiera, la integridad de los mercados y la capacidad de consumo de los individuos. Esto significa que el desarrollo de la "formalidad" del mercado resulta estratégica para el desarrollo de las operaciones que se realizan, así como el beneficio de la sociedad (CNBv, 2012).

La existencia de informalidad en los mercados crediticios es un problema grave para el desarrollo de las actividades de mercado financiero, en especial por la existencia de vicios o reglas externas que generan distorsiones. La informalidad opera por fuera de las instituciones legales o de sistemas normativos de regulación, donde los agentes realizan acuerdos privados. El principal problema son sus fallas: a) mayor riesgo y volatilidad; $b$ ) la existencia de usura; ${ }^{7}$ c) la explotación de los prestamistas sobre los prestatarios; d) la negligencia financiera y legal en el uso y consumo de los instrumentos, y e) la poca educación financiera de las personas. Además, alimenta los ciclos de poder y corrupción en las localidades, donde los agentes con cierto poder tienden a generar mercados ineficientes, monopolios, y sistemas semifeudales de tributación.

Los mercados informales surgen por las redes sociales, donde los individuos conocen a otros agentes que pueden satisfacer sus necesidades, con costos de transacción que se consideran no son tan elevados para ellos, dadas las circunstancias. La informalidad es un problema derivado de las fallas de mercado, por lo que el costo para el mercado y la sociedad son mucho mayores que su corrección mediante políticas públicas.

Los mercados financieros formales son aquellos que operan bajo un sistema normativo que regula su manejo, y que ofrecen a los demandantes de servicios financieros una amplia gama de posibilidades crediticias, de ahorro e inver-

7 Aunque la usura existe también en el mercado formal, se considera que es un delito que debe seguirse institucionalmente. Sin embargo, en los mercados informales, aunque sigue siendo un delito, se percibe como un costo adicional "aceptable". 
sión. El acceso a este mercado tiene la bondad de poder suavizar el consumo de los individuos a través del tiempo, así como ofrecer mejores oportunidades de desarrollo mediante la inversión. En el mercado formal existen relaciones de mercado que no dependen del cs con que cuenten los agentes, sino de la confianza y del poder de atracción de las instituciones bajo formas específicas de oferta de productos financieros. Esto garantiza mejores resultados para los demandantes de servicios, que se comprometen al pago único del costo de los servicios adquiridos. Sobre el crédito, es válido decir que los créditos formales e informales son sustitutos imperfectos entre sí, ya que ambos satisfacen la misma necesidad, pero no cuentan con la misma calidad (CNBV, ${ }^{8} 2012$; DiGiannatale et al., 2008).

El mercado de créditos es parte del mercado financiero, y es un bien negociado inter-temporal, donde también existen fallas de mercado. En el caso de México, su dinámica presenta diferentes distorsiones que han enviciado sus fallas y que han afectado la demanda de dichos servicios por gran parte de la población. ${ }^{9}$ Su función es redistribuir el ingreso, de manera que aquellos que poseen un ahorro puedan financiar a los que no. Es un servicio en el que se presta dinero a cambio de la devolución del préstamo, más una compensación. Como un bien físico, tiene una oferta y demanda. Existen instituciones que vigilan el funcionamiento de estas transacciones, de manera que no se permita el abuso (formalidad). Los prestatarios están constituidos por dos clases de agentes: a) instituciones financieras consolidadas (formales y anónimas) y $b$ ) agentes privados capitalizados (informales y conocidos, probablemente). Cada uno de estos oferentes está asociado a un nivel de riesgo, confianza y accesibilidad. Se considera que se puede hacer uso del cs para estudiarlo (DiGiannatale et al., 2008; Dornbusch, 2009 et al., 2001; Stiglitz, 1989).

De esta forma, el cs se vuelve una herramienta relevante en el análisis periférico del fenómeno de los mercados financieros, donde las personas tienden a arreglar la información que tienen a su alcance mediante sus relaciones con otros agentes, y que pueden ser ventanas directas de oportunidad, o bien, canales de transmisión de los pedazos de información con que no se cuenta.

Comisión Nacional Bancaria y de Valores.

9 Dejando aparte a grandes grupos comerciales, empresas y otros agentes con mayor capacidad de negociar y afrontar los altos costos de los servicios financieros en México. 


\section{METODOLOGÍA}

En este trabajo se construyeron dos grupos de modelos, lineales, para probar la sensibilidad de la demanda de crédito (DC) ante el uso de distintas variables relacionadas con el cs. Se construyeron dos grupos de modelos. En la primera parte se utilizaron cinco modelos que analizaron la dinámica interna de los mercados de servicios financieros, midiendo: Gasto en servicios financieros (M1), Tasa de ahorro bancario (M2), Tasa de crecimiento del crédito bancario (M3), Gasto en servicios financieros per cápita (M4), y el Crédito bancario per cápita (M5). El segundo grupo de modelos se centró en estudiar la magnitud con que cambiaban los modelos de DC y demanda de crédito per cápita (DCw) adicionándoles variables de Cs. Los modelos básicos están expresados en variables socioeconómicas formales, tratados directamente en el valor de sus registros.

La base de datos se construyó con datos oficiales de INEGI, Banxico, ${ }^{10}$ la ENIGH-2010, ${ }^{11}$ y la wvs-2010. ${ }^{12}$ Los datos se homogeneizaron al año base 2003, expresados en miles de pesos, en términos reales. Las variables de CS se trataron como promedios estatales, por lo que representan proporciones poblacionales de comportamiento.

\section{Supuestos}

Los supuestos que se hacen en esta investigación son: (a) los individuos están conscientes de la existencia de asimetría en la información, y son capaces (b) de resolver sus problemas mediante el uso de la información que obtienen de otras personas.

\section{Modelos}

Los modelos M1-M5 ${ }^{13}$ (1 a 5) son reconstrucciones del trabajo de Carvallo (2013), quien trataba de encontrar el grado de afectación de los mercados financieros debido al cs. Son cinco modelos (el modelo M3 fue hecho dos veces), que buscan encontrar la manera en que varían los resultados del modelo con la adición escalonada de variables.

\footnotetext{
10 Banco de México.

11 Encuesta Nacional Ingreso Gasto de los Hogares, 2010. INEGI, 2013.

12 World Values Survey (Encuesta Mundial de Valores), 2010.

13 Adicionando variables para su análisis comparativo.
} 
La segunda parte de los modelos se dedicó a establecer la relación directa entre los resultados de los modelos originales (DC y DCPC), y sus ampliaciones con variables de CS (DCw y DCpCw). Los modelos midieron la expansión de las ecuaciones de demanda para incluir las valoraciones de cs (Alarcón et al., 2003).

Hay trabajos que se han hecho para estimar el cs, y su efecto (De la Torre et al., 2011), basados en la distribución del ingreso (De la Torre, 2011; Neira, 2002; Alarcón y Bosch, 2003). El ingreso permite que las personas realicen actividades donde conocen y se relacionan con otras (como Putnam, 1993; citado por Boix y Posner, 1996), por lo que implica que el cs tiene un costo en sí mismo. Una aproximación posible es la que hace Carvallo (2013), valorando el efecto individual de algunas variables elegidas dentro de un sistema de modelos que describen las principales dinámicas del sistema financiero.

Alarcón et al. (2003) utilizaron datos de wvs en Chile para demostrar que se podía analizar el impacto de las políticas públicas en la sociedad mediante ecuaciones de cs, compuestas por: a) Civismo (pc), b) Confianza Institucional (ci), c) Participación Política (pp), y d) Variables socioeconómica (vse). La estructura que ellos propusieron fue:

$$
C S=g_{n}(x)=(v s e, p c, p p, c i)
$$

Para las DC, se usaron supuestos ordinarios sobre posibles variables que están asociadas a la demanda de capital (crédito), como ingreso, PIB ${ }^{14}$, y otros dispersores de riqueza, o determinantes de formación de capital. La ecuación de DC fue:

$$
D C=f(x)=f\left(\delta, \vartheta, \varepsilon, \mu, \varepsilon_{j}\right)
$$

Donde:

$\delta$ variables económicas; $\vartheta$ : apertura económica; $\mu$ : variables sociales; $\varepsilon$ : error.

Entonces, el modelo ampliado queda representado de la siguiente manera:

$$
D C_{\omega}\left(f(x) \wedge g_{n}(x)\right)=D C+C S+E
$$


Donde $D C_{\omega}$ es la Demanda de Crédito ampliada por los determinantes del cs, y E es un factor de Error estadístico. Por lo que el modelo general fue:

$$
D C_{\omega}=\beta_{0}+\beta_{i}(D C+C S)+\varepsilon_{z}
$$

\section{RESULTADOS Y DISCUSIÓN}

\section{Valores en México}

El análisis de valores fue útil para comprender qué es lo que piensa la población sobre sí misma; lo que espera de los demás, y de sí. Son valoraciones previas que se tienen antes de decidir una acción, e influyen en la economía, alterando su racionalidad. En este caso, también miden la confianza que tienen con su entorno, y lo que esperan de los demás. En este sentido, esta aproximación al cs les permite formular hipótesis previas sobre la conducta de otros, lo que perfila su análisis de riegos, beneficio-costo, y capacidad de acceso a los servicios de su interés. En términos económicos, les ayuda a definir sus expectativas racionales fuera y dentro del mercado. Véase gráfica 1.

En el caso del uso del sistema bancario, es el grado de confianza depositado en las instituciones financieras, además del nivel de uso de los servicios. Los datos muestran que el nivel es bajo para los estados del país, como lo dice Banamex ${ }^{15}$-unam (2008). ${ }^{16}$ Véase cuadro 2.

En el caso de la demanda de crédito el comportamiento es semejante al comportamiento del ahorro, aunque notoriamente más bajo. Aunque no se puede calcular la demanda de crédito informal (prestamistas, financieras locales, etcétera) debido a la escases de control y ausencia de información, se intuye que debe ser mayor. Véanse cuadro 3 y gráfica 2.

Detallando un poco más la percepción de valores de los mexicanos, existen patrones en la wvs ${ }^{17}$ en los que la percepción sobre el rumbo del país, y sobre los demás actores relevantes, son en promedio valoraciones negativas. ${ }^{18}$ En el caso de la mayoría de los Estados, la gente tiene la percepción de que el país no tiene el rumbo adecuado, así como demuestra una marcada desconfianza a la policía y al Sistema Judicial, al igual que a las instituciones bancarias.

\footnotetext{
15 Banco Nacional de México.

16 Universidad Nacional Autónoma de México.

17 Por el investigador del ITAM, Alejandro Moreno.

18 Muy bajas.
} 


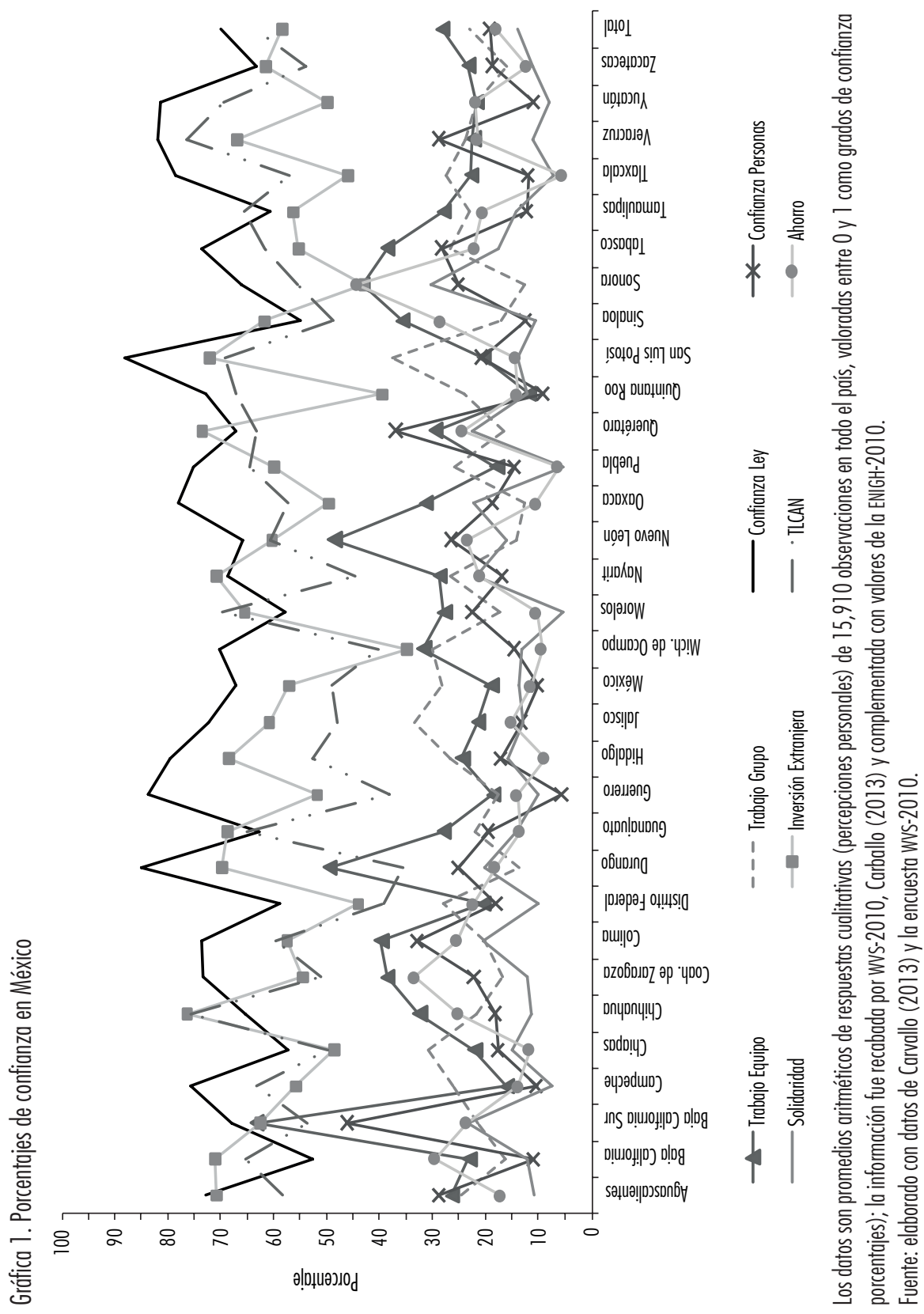




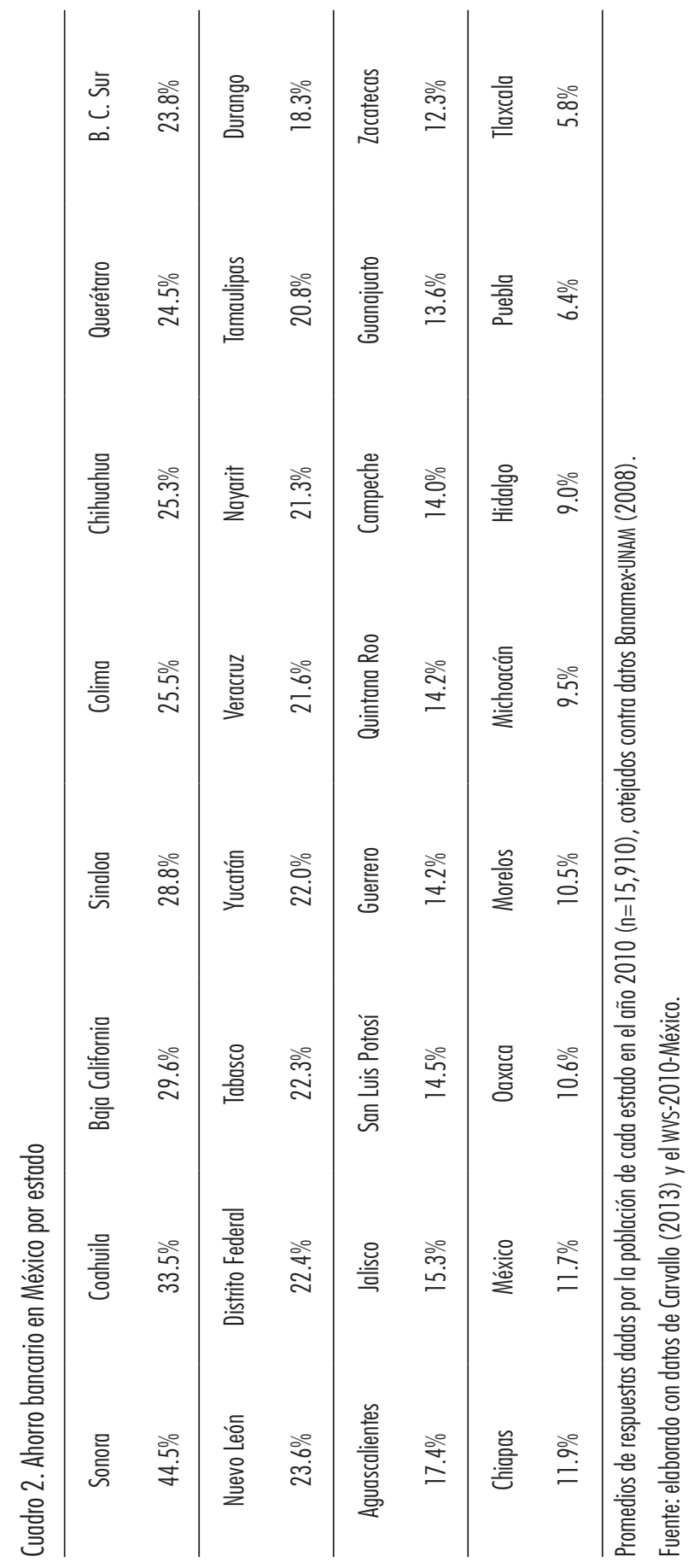




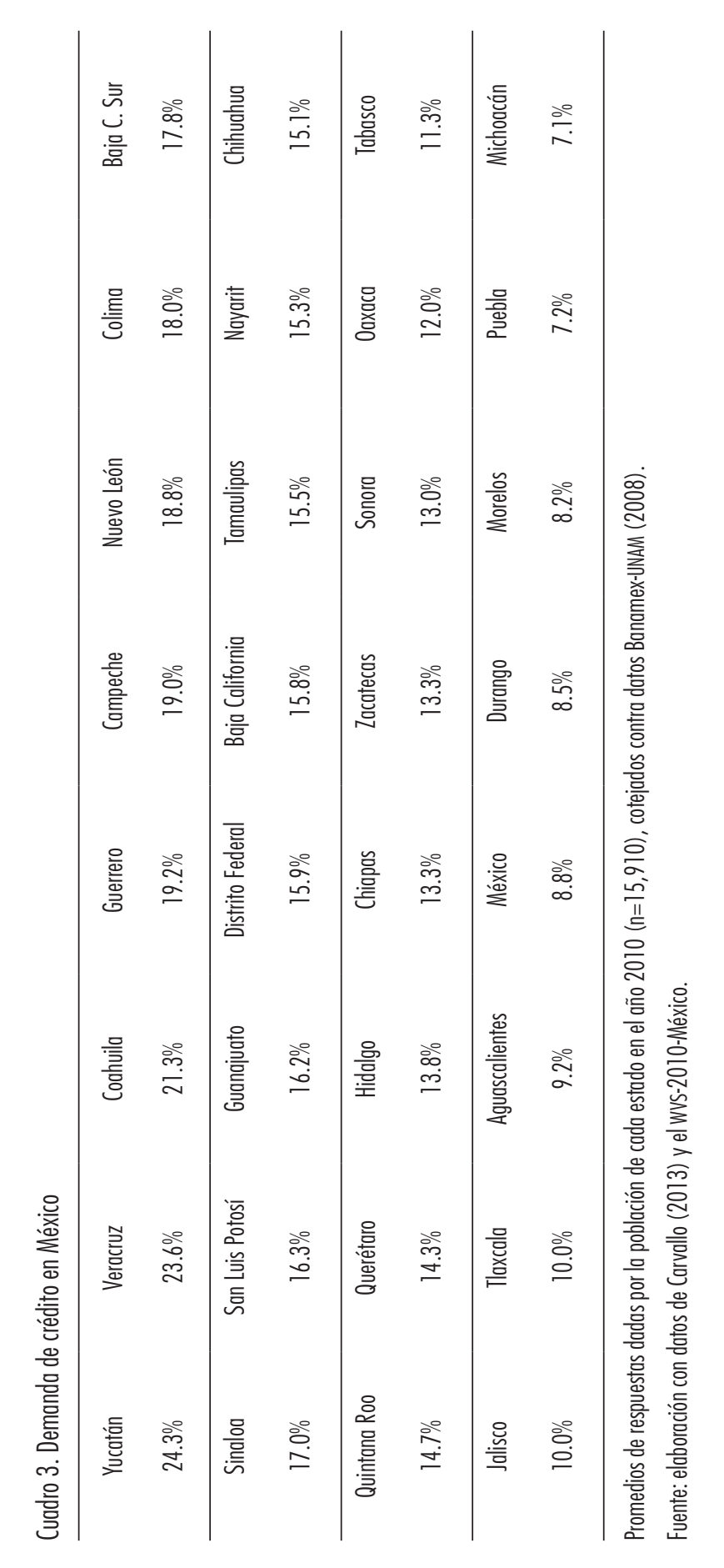




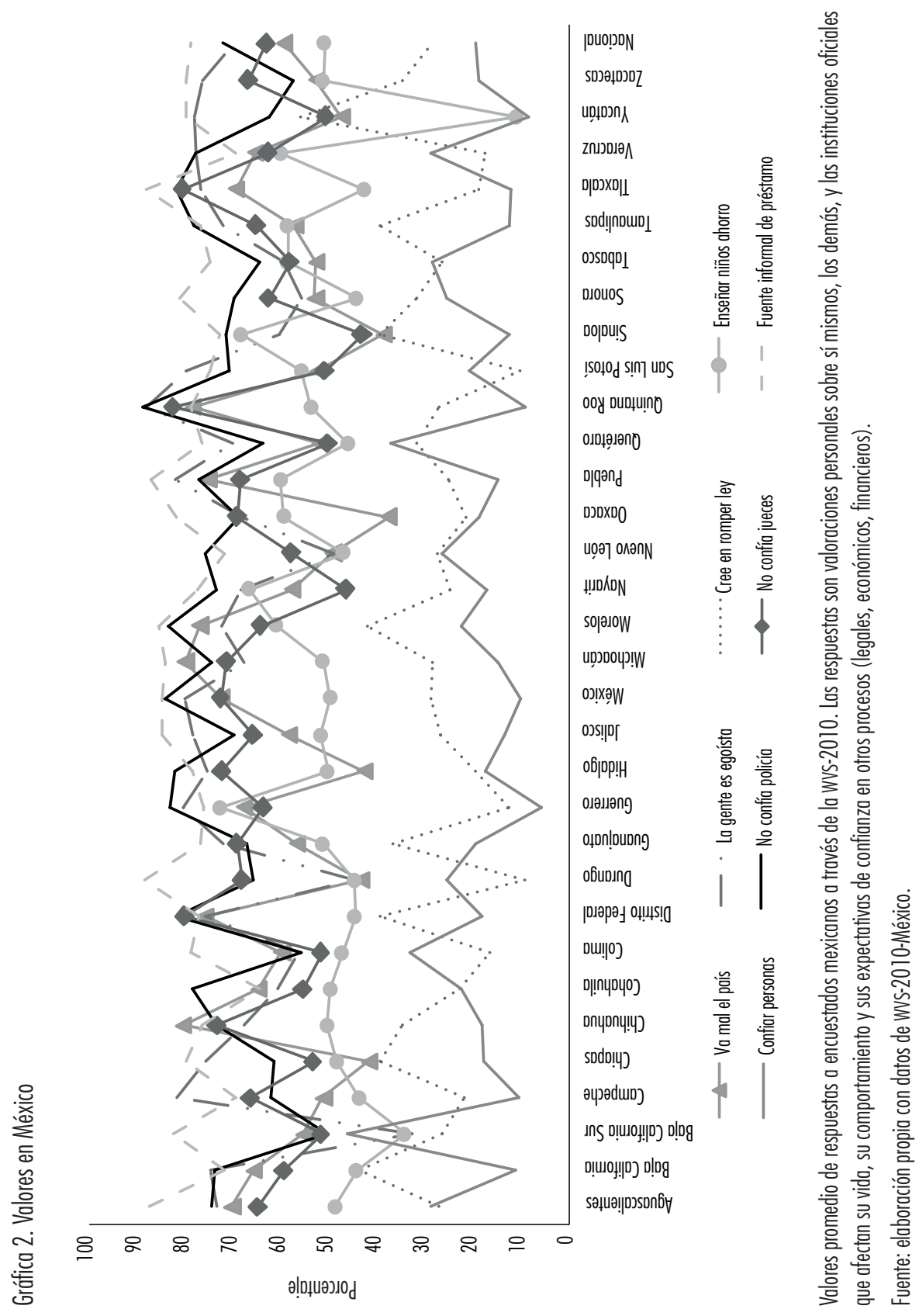


Esta información muestra que en México existe mucha desconfianza, que a su vez establece un clima de incertidumbre e inseguridad entre agentes. A nivel de las elecciones de los individuos, es previsible que éstos no confíen en el sistema formal ya que no se puede garantizar la seguridad de la propiedad privada, ni la integridad ética de los demás agentes (vistos como corruptos o negligentes). Todo esto sustenta la hipótesis de la fortaleza de las instituciones informales, que en México parecen ser más confiables pese al grado de volatilidad; parece preferible ese riesgo al de las instituciones formales, ya que se le ve menos problemático y peligroso, lo que genera cierto sentido de bienestar (aunque no tenga ningún fundamento real más que la percepción ${ }^{19}$ ).

En el caso de la corrupción son alarmantes los altos valores que obtiene, ya que sumados a las tendencias de los demás datos estudiados, muestran un mercado altamente contaminado, inseguro y con agentes que no confían entre sí. Esta clase de condiciones existentes en México permiten intuir los motivos por los que los agentes prefieren actuar por canales no institucionales, y que en el caso del sistema financiero nacional permite tener un contexto social más fuerte (informal), por lo que el desarrollo de herramientas y de resultados es pobre y deficiente. El análisis de estos valores es relevante porque permite comprender el porqué las personas toman decisiones que parecen irracionales dentro del mercado, pero que vistas en el contexto general en el que ocurren muestran una racionalidad justificada (externa al mercado).

\section{Modelos}

Se construyeron 11 modelos para analizar el fenómeno de la demanda de crédito en México, y estudiar así el comportamiento de la dinámica financiera en general. Los modelos fueron regresiones econométricas lineales simples, corridos en los programas IBM, ssps Statics Ver. 19 y sTATA/IC Ver. 12.

Los resultados obtenidos para los modelos corridos se muestran en el cuadro 4 .

De este cuadro se concluyó que la mayoría de los modelos en que fueron introducidas variables relacionadas al cs registraron un incremento visible en la representatividad (ajuste) estimado. Es necesario mencionar que una continua adición, superior a estos puntos en que se alcanzó un valor máximo, comenzó a provocar ligeras disminuciones.

19 Alimentado principalmente por el miedo a los procesos legales, y las constantes noticias de comportamiento corrupto e inmoral; adquirido en noticias y por terceros. 


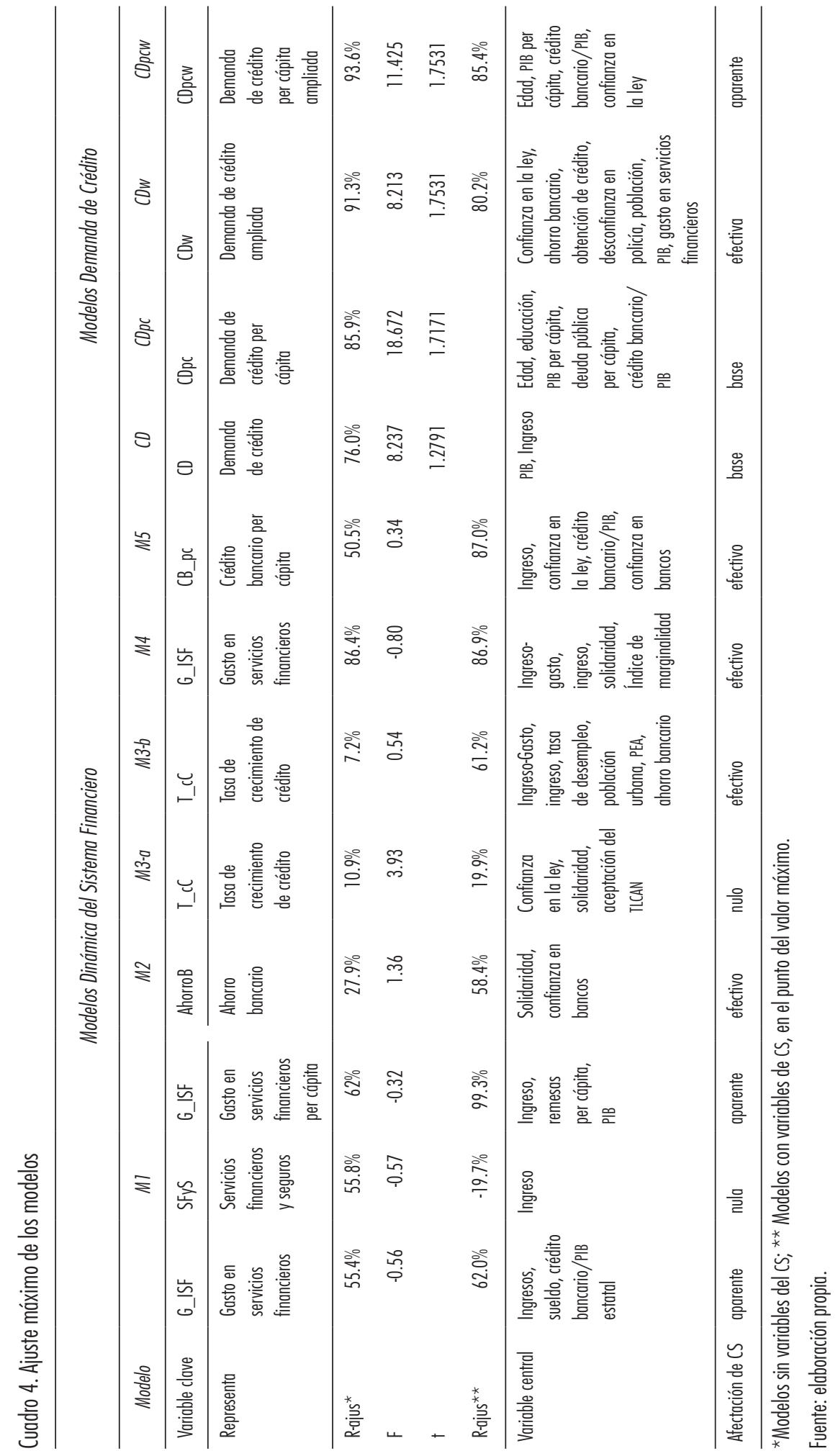


En M1 se obtuvo que la adición de variables de cs sí tuvo un efecto notorio en el Gasto en Servicios Financieros, ya que pasó de una significancia menor al $30.0 \%$ hasta $62.0 \%$, y hasta $93.0 \%$ en el per cápita. No obstante, en el caso de los servicios financieros y seguros, el resultado fue nulo, ya que el ajuste de la R-cuadrada fue bajo y con cambios de signos en los coeficientes. En el caso de M2, tuvo un cambio de 27.0 a $58.0 \%$ en su ajuste, adicionando las variables de cs; por lo que se concluyó que los valores sí afectan la manera en que las personas ahorran.

En el caso de los modelos M3 se tuvo que el modelo original M3-a fue insatisfactorio, ya que su nivel de ajuste siempre estuvo cercano al (-) $19.0 \%$. En este caso se decidió replantear los términos de la ecuación sustituyendo las variables de las unidades familiares por indicadores de capital. Esto elevó el ajuste de la R-cuadrada de M3-b a 61.2\%. En el caso de M4, los resultados mostraron que el cs sí afecta el nivel de ajuste, pero éste se encuentra muy vinculado con el ingreso. En el caso de M5 se estudió el crédito bancario per cápita, que respondió notablemente al Cs, ya que pasó de 50.0 a $87.0 \%$ con la inclusión de las variables.

En el caso de los demás modelos se obtuvo que las R-ajustadas fueron de: DC (76.0\%), DCpc (85.9\%), DCw (80.2\%) y DCpcw (85.4\%). Esto mostró que en el caso de la DC, al ser incluidas variables de valores, la representación del modelo se incrementó en casi 10 unidades porcentuales. En el caso del mismo modelo planteado en términos per cápita, la adición de variables mantuvo la representatividad en el $85.0 \%$, aunque con pequeñas variaciones; sin embargo, no la redujo, ni afectó demasiado a los coeficientes, por lo que se concluyó que el cs era importante, aunque no se pudiera establecer la relación con claridad.

Así, para los casos de interés, las variables relacionadas con la dispersión del ingreso (en términos per cápita) parecen mostrar que la hipótesis de que el CS se relaciona con el ingreso es acertada. Y que las variables de cs, de hecho, sí influyen en la toma de decisiones de los individuos. Si bien, la mayor parte de las variables importantes estuvieron caracterizadas por una percepción de confianza, éstas guardan una relación fuerte dentro de los procesos de elección. Para los demás casos, se infiere su importancia por el comportamiento de los resultados de los modelos. Véase cuadro 5.

Comparando el trabajo que han realizado otros investigadores, sobre la relación de cs y micro-finanzas, se tuvo que:

Uno de los resultados que se encontró fue el de que el planteamiento de Carvallo (2013) tenía sustento, aunque los datos con los que trabajaba no estaban tratados en forma correcta. Sus modelos fueron una aproximación 
Cuadro 5. Evaluación de las hipótesis de investigación

Hipótesis Conclusión

$\mathrm{HI}^{0}$ : El CS es un concepto que puede explicar las fallas del mercado.

H20: En México el CS tiene relación significativa con la demanda de créditos.

H30: Los estados de México responden al modelo planteado teóricamente que asocia el grado de demanda de crédito con el CS.

H40: Se puede establecer la naturaleza de la relación del CS con la DC.

H50: Los resultados de los modelos permiten realizar un análisis de política pública.

H60: Es posible construir ecuaciones de CS para los estados de la República mexicana.
Debido a los resultados del modelo, y las consideraciones teóricas, se acepta.

Debido a los resultados del modelo, y las consideraciones teóricas, se considera aceptable, pero no determinante.

Debido a los resultados del modelo, y las consideraciones teóricas, se acepta.

Debido a los resultados del modelo, y las consideraciones teóricas, se considera aceptable, pero no determinante.

Debido a los resultados del modelo, y las consideraciones teóricas, se acepta.

Se rechaza. No existe un parámetro desde el cual se puedan construir estos modelos. Sin embargo, puede construirse un Índice de CS , 0 utilizar las variables como ampliaciones de los modelos tradicionales.

Fuente: elaboración propia.

acertada, aunque fueron escasos en la cantidad de variables que podía utilizar, y la relación que éstas tienen con las decisiones de mercado. Es decir, si bien el cs afecta la demanda de servicios financieros, hay variables más sensibles, y que tienen justificación teórica (i.e., la confianza en los bancos, o el conocer medios de ahorro más benéficos [aunque sea polémico y debatible] que los formales). Entonces, el cs sí altera las decisiones de los individuos.

Al igual que DiGiannatale et al. (2008), los resultados se pudieron interpretar como una prueba positiva de que existe una relación entre el cs y el grado de desarrollo financiero en México, con base en el comportamiento de muchas de las variables seleccionadas, principalmente las que reproducen el valor que las personas asignan a la confianza (tanto en el ahorro bancario como en los mismos bancos, y la ley). La idea de que las personas hacen uso de sus relaciones para corregir la fallas de mercado basados en la información, parece ser cierta, ya que los individuos que respondieron que prefieren hacer uso de los medios informales de crédito y ahorro, encajan con las tasas de ahorro efectivo, de demanda de crédito (personas) y el uso del sistema financiero. La respuesta de los modelos, en términos de la posible influencia de las 
variables, se ajusta a esta idea. El planteamiento de la corrección de la falla de mercado causada por la mala información de Sokol (2007) parece tener sentido dentro de los resultados del modelos, ya que hay evidencia de que los agentes sí usan su cs para encontrar soluciones aceptables a los problemas dentro del mercado.

Aunque no se construyeron modelos como los de Alarcón y Bosch (2003), en los que se definía una ecuación específica de cs, se encontró que es posible el uso de variables que se aproximen al cs en forma directa. Lo más apropiado sería contar con un indicador como el que proponen De la Torre et al. (2011) para poder contrastarlo de forma modelada; pero no se tuvo un valor base de referencia para construir dicha ecuación. Es decir, no se pudo efectuar una reconstrucción mediante regresión, ya que no existió un dato original medido. No obstante, los resultados obtenidos se consideraron como aceptables. Se usó el principio de la Daga de Ockham, ${ }^{20}$ ya que hay evidencia empírica como para creer probables las hipótesis de esta investigación. De ser así, Espinoza y Rabi (2009) tuvieron razón al afirmar que el cs puede ser usado para evaluar, o reforzar esa evaluación, las políticas públicas, ya que al comprender el grado de afectación, y las preferencias en la estructura social, se pueden mejorar los programas existentes, o por aplicar, de cooperativas de ahorro y financiamiento, directamente en la población y mediante agentes que sean reconocidos y de confianza por la población objetivo. Todo esto fue congruente con la defensa de Boix y Posner (1996) sobre el trabajo de Putnam (1993).

No se usaron perfiles para especificar a los agentes que intervienen en el mercado, pero los resultados indicaron que los agentes tienen cierto poder en lo que ocurre dentro del mercado. Para comenzar, la mayor parte de los créditos pertenece a un sector muy reducido de la población, dado que la existencia de desigualdad (y por tanto de acceso al mercado) es muy marcada. En segundo lugar, los agentes que no participan en el mercado demuestran, de hecho, su poder, ya que generan una distorsión en el funcionamiento del mercado que no se puede ignorar; muchos de ellos simplemente por la apatía o la desconfianza en el sistema.

Es oportuno destacar que el uso de la wvs fue de gran importancia, pero no es la herramienta más adecuada. Es una encuesta demasiado general, y sus resultados pueden no ser los más recomendables para ser usados en los modelos que se puedan construir mediante regresiones. Sin embargo, pudieron usarse dentro de otras herramientas, y ser significantes. De momento no existe 
una metodología específica para comprobar esto, pero es posible que se puedan usar en modelos sobre el comportamiento de los agentes.

\section{CONCLUSIONES}

Aunque los resultados de los modelos planteados fueron positivos, no se puede ignorar el hecho de que los datos utilizados presentan problemas de compatibilidad, ya que la mayor parte de las variables de cs tienen expresiones porcentuales, que son las medias registradas por cada estado en el país. Por este motivo, no se concluye que sea una prueba definitiva de las hipótesis, sino que las conclusiones son inferencias basadas en la aparente posibilidad del efecto que causan, que dentro de este artículo se consideran como evidencia empírica aceptable para impulsar el debate de la cuantificación del cs.

Todas las hipótesis planteadas fueron evaluadas, cuando menos en los términos y consideraciones hechas. No fue posible construir ecuaciones por cada estado de su cs específico debido a que la información económica necesaria no se encuentra disponible de manera oficial.

Con base en lo anterior se pudo afirmar que el cs es un concepto complejo, pero que es una herramienta útil dentro del análisis económico; sobre todo en esos espacios grises en los que el mercado no es el espacio central de la toma de decisiones. Las personas usan el tráfico de información, que les ayuda a obtener beneficios a través de las relaciones que poseen. Es válido afirmar que es una especie de "capital", ya que se requiere un grado de inversión en él (actividades, tiempo y costos). En México el uso del sistema financiero es bajo respecto a la población que tiene algún ingreso y podría usarlo. Además, la educación financiera es insuficiente, y casi inexistente en áreas rurales, lo que contribuye a las distorsiones económicas y la marginación.

En términos generales, se consideró altamente probable que el cs se presente en las decisiones de los agentes que acuden a los mercados, eligiendo entre la formalidad y la confianza que tiene dentro de su red de relaciones. Esto ocurre en un espacio anterior al mercado, donde se resuelven algunos problemas relacionados con el acceso a medios de financiamiento, y de confianza en los servicios de instituciones formales.

El cs mostró en los resultados de los modelos que hay evidencia suficiente para afirmar que las variables de percepción de valores de las personas tienen un peso importante en su proceso de toma de decisiones. Quizá no se pueda inferir que la confianza a otros agentes y la preferencia de mecanismos, instituciones y personas sea determinante, o que sean la causa de la demanda de 
crédito, pero sí son uno de los factores explicativos del fenómeno; además, establecen un contexto sobre los procesos sociales asociados a los mercados que normalmente no se pueden identificar con modelos puramente cuantitativos.

El cs es un sistema multidireccional, con nódulos casi infinitos, en el que la información puede fluir de un punto a otro. Por esto, muchas de las distorsiones de la información asimétrica del mercado pueden corregirse. En qué medida el cs influye no es posible determinarlo con precisión, ya que su medición o construcción depende de las consideraciones teóricas que se haga en un inicio. En esta investigación el cs fue un factor que ayudó a los agentes a tomar decisiones fuera del mercado, por lo que puede que no sea necesario medirlo con precisión, sino identificar el peso e importancia que los agentes aplican a estas consideraciones exógenas al mercado mismo.

\section{BIBLIOGRAFÍA}

Alarcón Hein, Alex y José Luis C. Bosch (2003). Capital Social en Chile: avances sobre su formación y aplicación, Chile, CPU-Chile. 30 pp.

Boix, Carlos and Daniel Posner N. (1996). "Making Social Capital Works: a review of Robert Putnam's 'Making Democracy Works: Civic Traditions on Modern Italy', Paper no. 96-4, June, USA, The Weatherhead Center for International Affairs, Harvard University.

Bourdieu, P. (1985), "The Forms of Social Capital”, en J. Richardson (ed.), Handbook of Theory and Research for the Sociology of Education, NewYork, Greenwood.

Carvallo, Araceli (2013), Master Thesis. Social Capital. Attitude Towards Globalization and Financial Performance at Regional Level: The case of Mexico, Netherlands. MSc Economics y Business. Erasmus School of Economics, Erasmus University Rotterdam, $61 \mathrm{pp}$.

Comisión Nacional Bancaria y de Valores (CNBv). (2012/2013). Libro Blanco de Inclusión Financiera, México,CNBV.

De la Torre, Rodolfo and Patricia López-Rodríguez (2011), Closing the Gap: The Link between Social Capital and Microfinances Services, México, CIDE, $52 \mathrm{pp}$.

DiGiannatale, Sonia, Patricia López, y María José Roa (2008), Una introducción conceptual al desarrollo financiero, capital social y anonimidad: el caso de México, México, CIDE.

Dobruck Lowe, Jennifer (2012). Social Networks as an Anti-Poverty Strategy, USA, Crittenton Women's Union. 
Dornbusch, Rudiger, Stanley Fischer and Richard Startz (2009), Macroeconomia, 10a, China, McGraw-Hill.

Espinoza, Vicente and Violeta Rabi (2009), "Capital Social y civismo en las regiones chilenas", Informe del Proyecto Anillo SOC12, Chile, Facultad de Ciencias Sociales de la Universidad de Chile y Subsecretaría de Desarrollo Regional y Administrativo.

Mitchell K., Ronald, Bradley Agle R. and Donna Wood J. (1997), “Toward a Theory of StakeholderIdentification and Salience: Defining the Principle of Who and What Rreally Counts", Academic of Management Review, vol. 22, USA.

Neira, María Isabel (2002),. "Capital Social: concepto y estudio econométrico sobre el Capital Social en España”, Estudios Económicos de Desarrollo Internacional, vol 2, núm 2, España, AEEADE, Universidad de Santiago de Compostela, pp. 25-52.

Ríos-Figueroa, Julio y Alejandra Rios-Cázarez, (1999), "Capital Social y democracia: una revisión crítica de Robert Putnam”, Política y Gobierno, vol. VI, núm. 2. México, CIDE.

Sen, Amartya (2000), Desarrollo y libertad, México, Editorial Planeta.

Stiglitz, Joseph E. (1989), Markets: "Market failures, and development", The American Economic Review, vol. 79, núm. , USA, pp. 193-203.

Sokol Lederman, Natan (2007), "El papel del capital social en la corrección de problemas de información en los mercados financieros", Revista Venezolana de Análisis de Coyuntura, vol. XIII, núm. 2, julio-diciembre, Venezuela, Universidad Central de Venezuela, pp. 69-96. 
